\title{
Defect and Carrier Dynamics in Nanotubes under Electronic Excitations: Time-Dependent Density Functional Approaches
}

\author{
Yoshiyuki Miyamoto \\ Fundamental and Environmental Research Laboratories, NEC Corporation, \\ 34 Miyukigaoka, Tsukuba, 305-8501, Japan \\ y-miyamoto@ce.jp.nec.com
}

\begin{abstract}
One of challenging application of carbon nanotubes is nano-scaled electronic device, in which precise control of defects and carriers is required in analogy of silicon-based technology. In this work, we show that optical excitations can be promising tools to analyze and control defects in nanotubes being alternative to conventional heattreatments. We performed $a b$ initio calculations, which solve the time-dependent Schrödinger equations for electrons on real-time axis as well as classical Newton's equations of motions for ions. This method is also useful to investigate carrier dynamics under finite temperatures. These works were done under collaboration with Prof. Angel Rubio, Prof. David Tománek, Dr. Savas Berber, and Miss Mina Yoon.
\end{abstract}

\section{Introduction}

Carbon nanotubes have structures obtained by rolling up graphene sheets along with variety of chiral vectors [1]. The electronic structures of nanotubes are found to be dependent on their chiral vectors by theoretical works [2-5], which opened a door of nanotube application of electronics [6]. However, there remain a lot of hurdles to reach nanotube electronic devices superior to current silicon devices. In addition to contact problem between nanotubes and electrodes [7], some sort of imperfections in carbon nanotube should also be concerned. For example, conductivity of nanotube is reduced by a single vacancy according to the recent quantum conductance simulations $[8,9]$.

In this paper, we would like to demonstrate possibility of quality control of nanotube with use of electronic excitations as tools of defect identification and partial repair. These ideas were obtained by performing molecular dynamics (MD) simulations under electronic excitation. In the following sections, we will explain the computational method (in section 2), and detailed results (section 3). We also mention possibility to simulate charier dynamics. Finally, we will give concluding remarks.

\section{Computational Methods}

The MD simulations under electronic ground states are popular and done by many research groups, where eigenvalue problem of the quantum mechanics for electrons are solved at each time-step of ionic motion. The Car-Parrinello (CP) method [10] 
introduce fictitious equations of motion for electron wave functions throughout MD simulation, which is very useful to reach the geometric and electronic ground state as the converged solution for long-time simulations without diagonalizing very large matrix. On the other hand, MD simulation under electronic excitation is rather hard, since the order of the excited electron-level sometimes switch to those of full or empty level throughout the MD simulation. In such cases, solving eigenvalue problem or doing CP method causes miss-assignment of the electronic occupation and thus turns out to follow wrong potential energy surface (PES). To prevent these, Sugino and Miyamoto proposed MD simulation by solving the time-dependent Schrödinger equation for electrons [11]. Computer code for doing this type of simulation is named as FPSEID, which stands for First-Principles Simulation tool for Electron-Ion Dynamics.

\subsection{Time-dependent density functional theory for real-time axis}

In the method of Ref. [11], the time-dependent density functional theory (TDDFT) [12] is adapted. In contrast to conventional TDDFT approaches within a perturbation theory under external field oscillating with a given frequency, the present TDDFT treatment deals real-time dynamics of electrons, where a time evolution operator consisting of an exponential of the Kohn-Sham Hamiltonian $\left(H_{K S}\right)$ is directly applied to every electron wave functions. This method is coupled with the classical MD method, so we call present simulation as TDDFT-MD simulation.

Here, the $H_{K S}$ contains many non-commutable operators, i.e., kinetic energy operator, local Hartree-exchange-correlation potential $\left(\mathrm{V}_{\mathrm{HXC}}\right)$ of many-body system, and nonlocal parts of the pseudopotentials [13]. All of these operators are indispensable to express real materials and the number of operators is dependent on the system size and elements included in the system. Exponentials of $H_{K S}$ can be spited by a product of exponentials of individual operators according to the Suzuki-Trotter formula [14], which guarantees higher order of accuracy with respect to the time-step. Suppose that $H_{K S}$ is written as follows.

$$
H_{K S}=A_{1}+A_{2}+\ldots . .+A_{q-1}+A_{q}
$$

Here, $A_{1}$ through $A_{q}$ represent kinetic energy operator, nonlocal part of pseudopotentials, and local part of pseudopotential plus $\mathrm{V}_{\mathrm{HXC}}$. Number of operator $q$ is dependent on number of atoms included in the system. No matter what value the $q$ takes, the exponential of $H_{K S}$ can be approximated as following [14].

$$
e^{x H_{K S}} \cong e^{\frac{1}{2} x A_{1}} e^{\frac{1}{2} x A_{2}} \ldots . e^{\frac{1}{2} x A_{q-1}} e^{x A_{q}} e^{\frac{1}{2} x A_{q-1}} \ldots e^{\frac{1}{2} x A_{2}} e^{\frac{1}{2} x A_{1}} \equiv S_{2}(x)
$$

Here $x$ means -idt, and dt is interval of every time step. This splitting formula guarantees second order of accuracy with respect to $x$. By using the formula of equation (2), we can achieve higher order of accuracy as following [14].

$e^{x H_{K S}} \cong S_{2}\left(p_{1} x\right) S_{2}\left(p_{2} x\right) S_{2}\left(p_{3} x\right) S_{2}\left(p_{2} x\right) S_{2}\left(p_{1} x\right)$ 
Here $p_{1}, p_{2}$, and $p_{3}$ are c-numbers given as follows [14].

$$
p_{1}=p_{2}=\frac{1}{4-4^{\frac{1}{3}}}, P_{3}=4-4 p_{1}
$$

Next, we must know how to operate the exponential of individual operators. Since we express the wave functions as an expansion of plane waves, kinetic energy operator and $\mathrm{V}_{\mathrm{HXC}}$ can be operated on reciprocal and real spaces, respectively. Thus operations of these exponentials are straightforwardly treated.

Meanwhile, operation of exponentials of non-local pseudopotentials seems rather complicated. Fortunately, the presently used pseudopotentials [13] have the separable form [15] as following.

$$
V_{\tau l m}^{p s}(r)=\frac{V_{\tau l}^{p s}\left|P_{\tau l m}><P_{\tau l m}\right| V_{\tau l}^{p s}}{<P_{\tau l m}\left|V_{\tau l}^{p s}\right| P_{\tau l m}>}
$$

Here, $\mathrm{t}$ means atomic site, $l$ and $m$ mean angular momentum and magnetic quantum numbers. $\mid P>$ is a projection operator of atomic pseudo-orbital. After an algebra using Taylor's expansion, we obtain exponential of equation (5) as following [11].

$$
e^{x V_{\tau l m}^{p s}(r)}=1+\frac{V_{\tau l}^{p s}\left|P_{\tau l m}>\left\{e^{x \frac{\left\langle P_{\tau l m}\left|V_{\tau l}^{p s} V_{\tau l}^{p s}\right| P_{\tau l m}>\right.}{<P_{l l m}\left|V_{\tau l}^{p s}\right| P_{\tau l m}>}}-1\right\}<P_{\tau l m}\right| V_{\tau l}^{p s}}{<P_{\tau l m}\left|V_{\tau l}^{p s} V_{\tau l}^{p s}\right| P_{\tau l m}>}
$$

Further techniques for the numerical stability, like as smoothing of charge and potential in the reciprocal space, and as extrapolation of time-evolving potential are described in Ref. [11].

Meanwhile, atoms are moved by the Newton's equations of motion according to the computed Hellmann-Feynman forces. This treatment is valid unless the simulation significantly deviates from the true adiabatic PESs. The deviation can be recognized by appearance of off-diagonal elements of the Kohn-Sham Hamiltonian with respect to the time-evolving wave functions of electrons [11].

\section{Results}

When a device size is reducing to nano-scale, existence of defect causes the strong scattering of the carriers and may trigger the structural degradation of the device. In analogy of conventional semiconductor technology, some methods of defectidentification are required to make a feed-back to growth and processing conditions of nanotubes. In this section, we introduce a promising way of defect identification and repair method suggested by the present TDDFT-MD simulation. 


\subsection{Identification of the Stone-Wale defect}

Stone-Wale (SW) defect is a sort of structural defect in honeycomb network of graphitic material [16] obtained by in-plane rotation of a C-C bond by 90 degree. The presence of the SW defect is predicted to affect the conductivity of nanotube by firstprinciples simulation [9]. Although the presence of the SW defects is widely expected, experimental identification of this kind of defect has not been reported. Transmission electron microscope (TEM) is a useful tool to analyze structural defects such as pentagon and heptagon on nanotubes [17], because presence of this type defect causes bending of nanotube. However, the SW defect does not cause the bending, so it is not feasible to identify the SW defects by TEM. Meanwhile, couple of theoretical works $[18,19]$ predicted the possibility of monitoring the SW defect by scanning tunneling microscopy (STM). Yet, it should be a rare event to take an image of SW defect by STM since the scanning of entire region of nanotube is very timeconsuming.

We here propose a method of identification of the SW defect, which is a combination of optical excitation and measurement of the induced vibration. Figure 1 shows our simulated SW defects on the very thin nanotube with diameter of 4 ?. In the left panel of Fig. 1, the C-C bond in the center of the SW defect is parallel to the tube axis, while in the right panel the $\mathrm{C}-\mathrm{C}$ bond is at an angle.
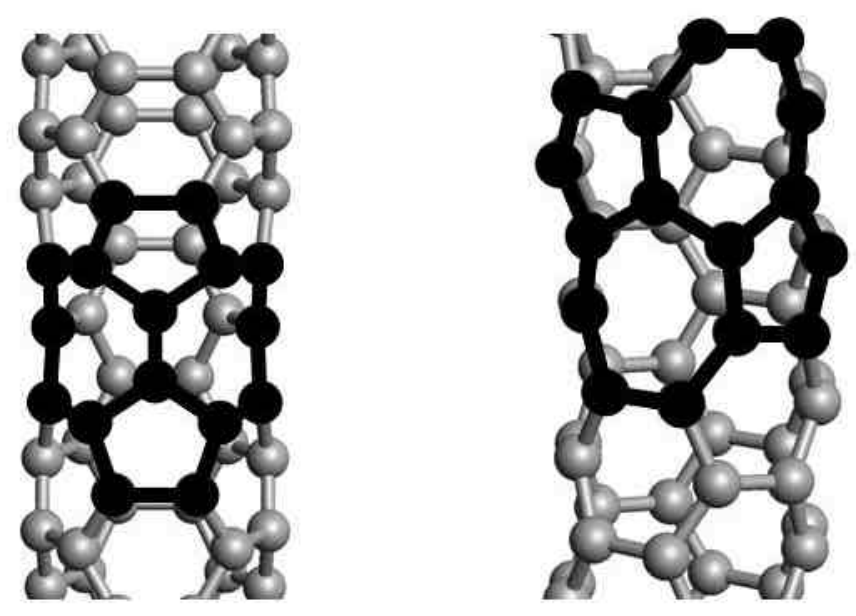

Figure 1: Stone-Wales defects on $(3,3)$ nanotubes. The black circles and lines denote the pentagon-heptagon pairs associated with the Stone-Wales defect. The C-C bond centered at the SW defect is parallel (left) or at an angle (right) with respect to the tube axis.

In both cases, stretching motion of the $\mathrm{C}-\mathrm{C}$ bond centered at the $\mathrm{SW}$ defect can be initiated by optical excitation. In case of SW defect at the left of Fig. 1, we found two possible optical excitations for $h ?=0.6 \mathrm{eV}$ and $6.0 \mathrm{eV}$. According to the TDDFT-MD 
simulation, we found induction of the vibration with a frequency of $1962 \mathrm{~cm}^{-1}$. While the difference between these vibrations with different excitation energies is their amplitudes. (See the bottom of Fig. 2.) The larger amplitude in higher excitation may be understood by multiple excitation of phonon, but this cannot be confirmed since the present simulation treats ionic motion with a classical manner.

On the other hand, so far, we found only one optical excitation $(h ?=1.0 \mathrm{eV})$ that induces the localized vibration mode in the SW defect as shown in upper of Fig. 2. This part denotes rather lower frequency compared to the case show in the bottom. The lower frequency is probably due to the bond length difference between these two SW defects, which is due to curvature of nanotube wall. Therefore, we expect the difference will disappear with increased tube-diameter.

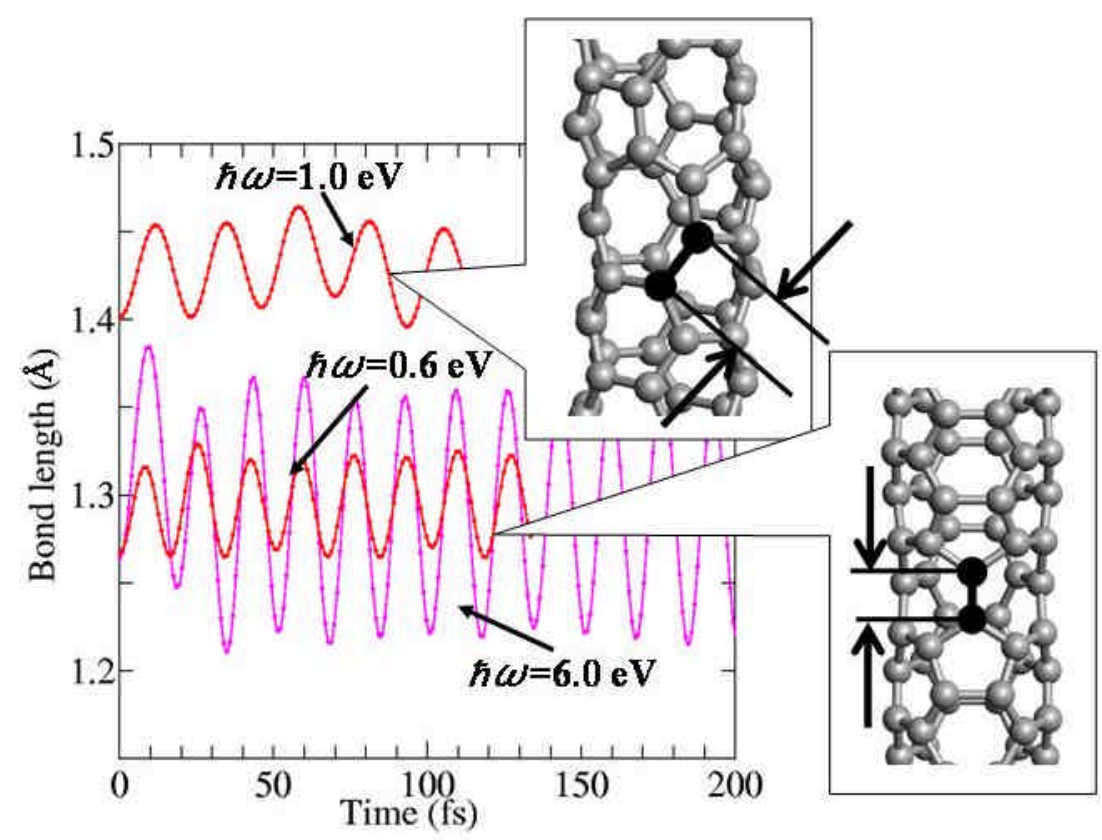

Figure 2: Time evolution of the C-C bond length centered at the Stone-Wales defect for the left panel (lower) and the right panel of Fig. 1. Corresponding photo-excitation energy is also denoted.

We have shown the possibility of experimental identification of SW defects in nanotube. Computational results show strong enhancement of the local vibration mode at the defect by optical excitation. Comparison of vibrational spectroscopy with and without excitation can highlight the existence of the SW defects. 


\subsection{Ultrafast self healing of single vacancy}

Single vacancy in nanotubes is also expected to exist as well as SW defect. Reduction of conductance due to presence of the single vacancy was theoretically predicted [9]. Furthermore, presence of single vacancy also causes oxidation of nanotube, which is reported from simulation [20] and experiment [21], those facts strongly indicate degradation of defective nanotube by oxygen. As shown in the left most panel of Fig. 3 , creation of a single vacancy causes creation of three dangling bonds of the sorbital. Termination of these three dangling bonds are however hard even when $\mathrm{H}$ atoms are used because of a narrow space of the vacancy.

We here propose a method to reduce number of the dangling bond from three to one. The idea is again electronic excitation which can partially stabilize the single vacancy in a very short time. Figure 3 shows procedure of the partial healing upon optical excitation from a defect-localized state into an excited state which is also localized at the defect.

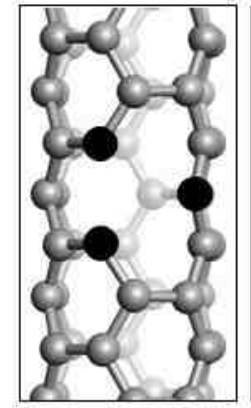

0 fs

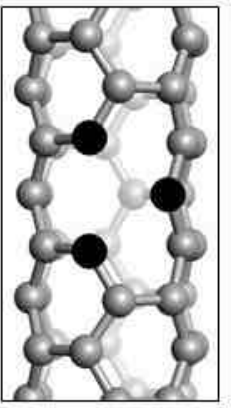

$50 \mathrm{fs}$

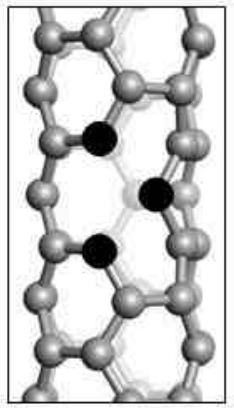

$100 \mathrm{fs}$

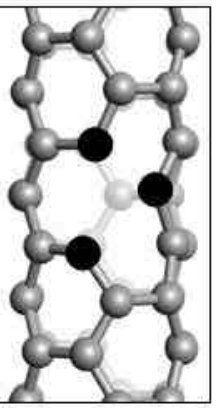

$150 \mathrm{fs}$

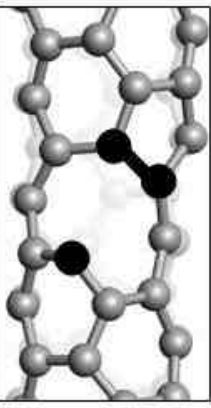

200 fs

Figure 3: Self-healing procedure of a single vacancy of a $(3,3)$ nanotube induced by the optical excitation. The black circles at each panel show three carbon atoms neighboring to a single vacancy. In the panel most right, newly formed $\mathrm{C}-\mathrm{C}$ bond is denoted by a thick line.

Surprisingly, the healing process requires a very short time-constant of $200 \mathrm{fs}$. On the electronic ground state, the activation barrier for this selfhealing is $0.3 \mathrm{eV}$. Therefore, thermal processes must take the time-constant of ps. One big advantage of ultrafast healing by the photoexcitation is reduction of probability of being attacked by any contaminants during the healing.

\subsection{Possibility of simulating the carrier dynamics}

Present TDDFT-MD simulation expresses time-evolutions of both electrons and ions. Throughout the electron-time-evolution, we re-compute the charge density from time- 
evolving wave functions, so charge density also evolves with respect to time as well as $\mathrm{V}_{\mathrm{HXC}}$ of DFT. Therefore, dynamical electron-electron interaction should be expressed within DFT. Meanwhile, ionic motion is also included so electron-phonon interaction should be expressed within classical manner.

We are now investigating hot-carrier decay dynamics in a perfect $(3,3)$ nanotubes. We found the $\mathrm{p}$ and $\mathrm{p}^{*}$ excitation at the G point as an optically allowed transition. By subtracting the total energy between the promoted state and the ground state, excitation energy is obtained as around $7 \mathrm{eV}$. The $(3,3)$ nanotube was experimentally examined [22], but the corresponding optical spectroscopy does not include the transition at the G point. We suspect that the considered excitation has a short lifetime and the TDDFT-MD can prove this. Now, this simulation is on-going.

\section{Concluding remarks and acknowledgments}

Motivating defect control in nanotube, we proposed optical excitation as alternative to conventional thermal processes. Defect identification and stabilization have been proposed. We expect these simulations will stimulate experimental works. Possibility of real-time dynamics of hot-carriers in nanotubes is discussed, and calculation examining this idea is now on going.

Part of present work were done by using the SX4 Supercomputer system at NEC Tsukuba Laboratories, the SX5 Vector-parallel system at NEC Fuchu Plant, and the Earth Simulator in Yokohama.

\section{References}

[1] S. Iijima, "Helcal microtubles of graphitic carbon", Nature (London), Vol. 354, pp56-58 1991.

[2] N. Hamada, S. Sawada, and A. Oshiyama, "New One-Dimensional Conductors: Graphitic Microtubules”, Physical Review Letters, Vol. 68, pp1579-1581, 1992.

[3] R. Saito, M. Fujita, G. Dresselhaus, and M. S. Dresselhaus, "Electronic Structure of Graphene Tubules base on C60", Physical Review B, Vol. 46, pp1804-1811, 1992.

[4] C.T. White, D. H. Robertson, and J. W. Mintmire, "Helical and rotational symmetries of graphitic tubules", Physical Review B, Vol. 47, ppR5485-R5488 1993.

[5] K. Tanaka, K. Okahara, M. Okada, and T. Yamabe, "Electronic property of bucky-tube model", Chemical Physics Letters, Vol. 191, pp469-472 1992.

[6] See, for example, S. J. Wind, J. Appenzeller, and Ph. Avouris, "Lateral Scaling in Carbon-Nanotube Field-Effect Transistors", Physical Review Letters, Vol. 91, pp583011-58301-4, 2003.

[7] S. Heinze, J. Tersoff, R. Martel, V. Derycke, J. Appenzeller, and Ph. Avouris, "Carbon Nanotubes as Schottky Barrier Transistors", Physical Review Letters, Vol. 89, pp1068011-106801-4, 2002.

[8] M. Igami, T. Nakanishi, T. Ando, "Conductance of Carbon Nanotubes with a Vacancy", J. Phys. Soc. Japan, Vol. 68, pp716-719, 1998.

[9] H. J. Choi, J. Ihm, S. G. Louie, and M. L. Cohen, "Defects, Quasibound States, and Quantum Conductance in Metallic Carbon Nanotubes", Physical Review Letters, Vol. 84, pp2917-2920, 2000.

[10] R. Car and M. Parrinello, "Unified Approach for Molecular Dynamics and Density Functional Theory", Physical Review Letters, Vo. 55, pp2471-2474, 1985.

[11] O. Sugino and Y. Miyamoto, "Density functional approach to electron dynamics: Stable simulation under a self-consistent field", Physical Review B, Vol. 59, pp2579-2586, 
1999; ibid, "Errata", Vol. 66, pp89901-1(E)-89901-1(E), 2002.

[12] E. Runge, and E. K. U. Gross, "Density Functional Theory for Time-Dependent Systems", Physical Review Letters, Vol. 52, pp997-1000, 1984.

[13] N. Troulleir, and J. L. Martins, "Efficient pseudopotentials for plane-wave calculations", Physical Review B, Vol. 43, pp1993-2006, 1991.

[14] M. Suzuki, "General Nosymmetric Higher-Order Decomposition of Exponential Operators and Symplectic Integrators". Journal of Physical Society of Japan, Vol. 61, ppL3015-3019, 1992; M. Suzuki and T. Yamauchi, "Convergence of unitarity and complex decompositions of exponential operators", Journal of Mathematical Physics, Vol. 34, pp4892-4897, 1993.

[15] L. Kleinmann and D. M. Bylander, "Efficacious Form for Model Pseudopotentials", Physical Review Letters, Vol. 48, 1425-1428, 1982.

[16] A.J. Stone and D.J. Wales, "Theoretical studies of icosahedral $\mathrm{C}_{60}$ and some related species". Chemical Physics Letters 128, pp501-503, 1986.

[17] S. Iijima, T. Ichihashi, and Y. Ando, "Pentagons, heptagons and curvature in graphite microtubule growth", Nature (London), Vol.356, pp776-777, 1992.

[18] A. Rubio, 'Spectroscopic properties and STM images of carbon nanotubes", Applied Physics A: Matererial Science and Process, Vol. 68, pp275-282, 1999.

[19] D. Orlikowski, M.B. Nardelli, J. Bernholc, and C. Roland, "Theoretical STM images and transport properties of native defects in carbon nanotubes", Physical Review B, Vol.61, pp14194-14203, 2000.

[20] M. S. Mazzoni, et al., "Energetics of the oxidation and opening of a carbon nanotube", Physical Review B, Vol. 60, ppR2208-22211, 1999.

[21] A. Kuznetsova, "Oxygen-Containing Functional Groups on Single-Wall Carbon Nanotubes: NEXAFS and Vibrational Spectroscopic Studies", Journal of American Chemical Society, Vol. 123, pp10699-10704, 2001.

[22] Z. M. Li, et al., "Polarized Absorption Spectra of Single-Walled 4 A Carbon Nanotubes Aligned in Channels of an AlPO4-5 Single Crystal", Physical Letters, Vol. 87, pp127401-1-127401-4, 2001. 